\title{
Innovative Partnership between a Rural Mental Health Center and Community Pharmacy: Integration of a Mental Health Pharmacist
}

ShuYing Ng, PharmD ${ }^{1,2}$; Robert Buckner Jones, $M D^{1}$; Laura Schwartzwald, BSPharm²; Meena Murugappan, PharmD;

Amy Pittenger, PharmD, MS, PhD ; Kevin Edwards, PhD, LP'; Randall Seifert, PharmD ${ }^{3}$

${ }^{1}$ Northern Pines Mental Health Center, Brainerd, MN; ${ }^{2}$ GuidePoint Pharmacy, Brainerd, MN; ${ }^{3}$ University of Minnesota, College of

Pharmacy, Minneapolis, MN

\begin{abstract}
Purpose: The purpose of this article is to describe how an innovative partnership between a rural community mental health center, community independent pharmacy and College of Pharmacy and integration of a mental health pharmacist lead to identification of medication therapy problems (MTP's) and interprofessional team partnerships with center mental health professionals.

Methods: A contractual arrangement was initiated between Northern Pines Mental Health Center (NPMHC), GuidePoint Pharmacy Services GPS) and the University of Minnesota College of Pharmacy (UMN CoP) to place a PGY1 resident at NPMHC. The resident was assigned to work closely with the Chief Medical Officer and provide initial comprehensive medication management (CMM) services to individuals who were enrolled in Assertive Community Treatment (ACT). A retrospective chart review was conducted to evaluate the impact of services provided. Patient inclusion criteria included ACT enrollees 18 years or older, a diagnosis of SPMI, taking at least one psychotropic medication, and participation in at least one resident-led CMM visit. Additional findings included the relationship between the pharmacist, the psychiatric physician, and other members of the ACT team. Descriptive statistics were used to document the findings. Findings: $N=30$ met the inclusion criteria: 18 males and 12 females, age ranged from 24 - 69 with average of 44 years old. 110 MTPs were identified ranging from no MTPs to 10 MTPs per patient, with a mean of 4 MTPs/patient. There was an uneven distribution of MTPs between psychiatric and medical conditions, with a disproportionately high occurrence of "Needs Additional Drug Therapy" in medical conditions and "Adverse Drug Reaction" in psychiatric conditions. In addition, the services were valued by members on the ACT team.

Conclusion: Rural residents with SPMI in intensive community treatment have complex medication needs that require the training and skills of a clinical pharmacist. Despite the inclusion of a medication list as part of the ACT fidelity standards MTPs may go unrecognized and unresolved without the services of a clinical pharmacist conducting CMM. The pharmacist and psychiatric physician formed a collaborative partnership to address medication issues. We conclude that there is a need for integrating clinical pharmacist services into rural mental health centers.
\end{abstract}

Key Words: rural mental health, psychiatric pharmacy, clinical pharmacist services, Comprehensive Medication Management, Assertive Community Treatment

The national burden of mental health and substance use disorders is significant and rural areas are affected disproportionately. Access to care is a nationally recognized barrier to receiving mental health services and this problem is most prominent among rural communities. The U.S. Health Resources \& Services Administration (HRSA) reported that there were 3,669 mental health Health Professional Shortage Areas (HPSAs) containing almost 91 million people in the past year. It would take 1,846 psychiatrists and 5,931 other practitioners to fill the needed slots ${ }^{1}$. For instance, in Minnesota (MN) all regions other than the Twin Cities metro area have been designated as mental health HPSAs. Another concern is that the aging of existing mental health workforce. The median age of U.S. psychiatrists is 55.7 years, with $46 \%$ over the age of 65 years. The same trend is seen among social workers ${ }^{1}$ and addiction treatment service providers, exacerbated by high workforce turnover rate ${ }^{2}$. It is vital to fortify the existing behavioral health workforce to extend access to quality care.

Corresponding author: ShuYing $\mathrm{Ng}$, PharmD

Phone: 218-829-3235, Fax: 218-829-1368

Email: shuyingng@npmh.org
Another challenge in providing optimal care for patients living with mental health and substance use disorders is the fragmentation of care. Adults living with a serious mental illness (SMI) are at increased risk for having comorbid chronic medical conditions. On average persons with SMI die 25 years earlier, due to these treatable medical conditions ${ }^{3}$. These patients are often found to be affected by a significant health disparity due to the continued lack of adequate integration of care between psychiatry and primary care. It has been well-established that these patients often fall through the cracks of metabolic monitoring, not only because of consequences of their mental illness leading to missed appointments and lower adherence to medications, but also due to the lack of regular connection with a primary care provider ${ }^{4}$.

Pharmacists are well positioned to bridge primary care and psychiatric care and also fill the rural workforce shortage in a cost effective manner. Given that a majority of patients with mental illnesses have several comorbid medical conditions, complex medication regimens with significant negative side effect profiles and poor treatment adherence, pharmacists can help in optimizing outcomes ${ }^{5}$. Comprehensive clinical pharmacist activities include medication evaluation and monitoring, 
optimization of medication regimens, therapeutic recommendations, laboratory test ordering and interpretation, prescribing authority under collaborative protocols with physicians, patient education, education of behavioral health staff including prescribers, and consultation services ${ }^{6}$. There have been several past examples of successful incorporation of clinical pharmacy services within mental health and substance use

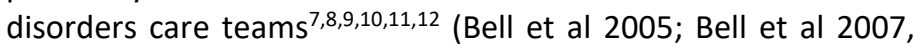
Chen 2001; Chen 2007; Finley et al 2003; Richardson et al 2014). Services provided by a psychiatric pharmacist working within a health care home model resulted in a 2.8:1 return on investment ${ }^{13}$. Other studies have proven the value of a pharmacist by showing an improvement in adherence to metabolic monitoring guidelines for patients receiving secondgeneration antipsychotics ${ }^{14}$, achievement of response to pharmacotherapy in patients with comorbid depression and diabetes ${ }^{15}$, improvement in functioning and decreased overall severity of illness ${ }^{16}$, and decreased hospital readmission within 30 days ${ }^{17}$.

To our knowledge, there have been no reports or studies of partnerships that have integrated a clinical pharmacist into rural community mental health centers. Therefore, we describe the unique partnership and care model implemented in a rural Minnesota mental health center.

\section{Methods}

\section{Care Model Partnership and Implementation}

Recognizing a need for more accessible, comprehensive, affordable, and high quality psychiatric care in rural Minnesota, the University of Minnesota College of Pharmacy spearheaded an innovation in pharmacy practice to address this need. The College started a post-doctoral Postgraduate Year-1 (PGY-1) Pharmacy Residency program with a concentration in mental health by partnering with GuidePoint Pharmacy and Northern Pines Mental Health Center (NPMHC) in Brainerd, MN. Through this program, a PGY-1 pharmacy resident was incorporated into the NPMHC Assertive Community Treatment (ACT) multidisciplinary care team.

The pharmacy resident (SN) functioned as a member of the ACT team and traveled to patients' homes to provide Comprehensive Medication Management (CMM) services, in addition to pointof-care-testing services as indicated. Provision of CMM is in adherence to the Pharmacists' Patient Care Process as defined by the Joint Commission of Pharmacy Practitioners (JCPP) where each medication (prescription or over-the-counter), nutritional supplement, or other treatment is assessed for appropriate indication, effectiveness, safety concerns, and barriers to adherence. The pharmacy resident worked with the patients and their respective NPMHC and non-NPMHC providers, which includes primary care as well as other specialists to collaboratively manage the often complex medication regimens in this population. Resident supervision was consistently provided by clinical preceptors at GuidePoint Pharmacy and College of Pharmacy.

\section{Assessment of Care Model}

A retrospective chart review was conducted by the pharmacy resident and collaborating psychiatric physician. Inclusion criteria: ACT program enrollees 18 years and older, diagnosis of a Serious and Persistent Mental Illness (SPMI), taking at least one psychotropic medication, and participation in at least one pharmacy resident-led CMM visit from July 2016 to April 2017. Primary outcome measures are the number and type of MTPs. Secondary outcomes investigated are the number of MTPs in psychiatric and medical illnesses. Additional care model anecdotal results including provider well-being and job satisfaction were recorded. Deidentified data was recorded with Microsoft Excel and stored securely in agency-issued secured laptop. The analyses of the deidentified data were exempt from consent by the institutional review board of the University of Minnesota (ETHOS ID: 152).

\section{Results}

Thirty patients met the inclusion criteria: 18 males and 12 females with an average age of 44 years old (ranged from 23 - 69 years old). Nineteen patients had a diagnosis of schizophrenia, eight with bipolar disorder and three with major depressive disorder (Table 1). All patients had comorbid medical or mental illnesses including substance use disorders and high use of tobacco and caffeine (Table 1).

Table 1. Diseases Background

\begin{tabular}{|lc|}
\hline Principal Psychiatric Diagnosis & N (\%) \\
\hline Schizophrenia & $19(63)$ \\
Bipolar Disorder & $8(27)$ \\
Depression & $3(10)$ \\
\hline Comorbid Conditions & \\
\hline Depression & $6(20)$ \\
Anxiety & $12(40)$ \\
Substance Use Disorder & $8(27)$ \\
Hypertension & $6(20)$ \\
Diabetes Mellitus & $7(23)$ \\
Dyslipidemia & $9(30)$ \\
Pain & $10(33)$ \\
COPD & $3(10)$ \\
\hline Lifestyle & \\
\hline Tobacco & $23(77)$ \\
Alcohol & $11(37)$ \\
Caffeine & $27(90)$ \\
\hline Illicit Drugs & $6(20)$ \\
\hline
\end{tabular}

Using standard pharmacist CMM process, 110 medication therapy problems (MTPs) were identified ranging from no medication therapy problems per patient to as many as 10 
medication therapy problems per patient, and a mean of 4 MTPs per patient. One patient did not have any identified MTPs during this specified timeframe. The most frequent MTP was Needs Additional Drug Therapy. This was due to a number of factors but most often related to a need for preventive immunizations which had not been administered and the need for cardiovascular event preventative therapy (Figure 1).

Figure 1. Types of Medication Therapy Problems

\section{Medication Therapy Problems}

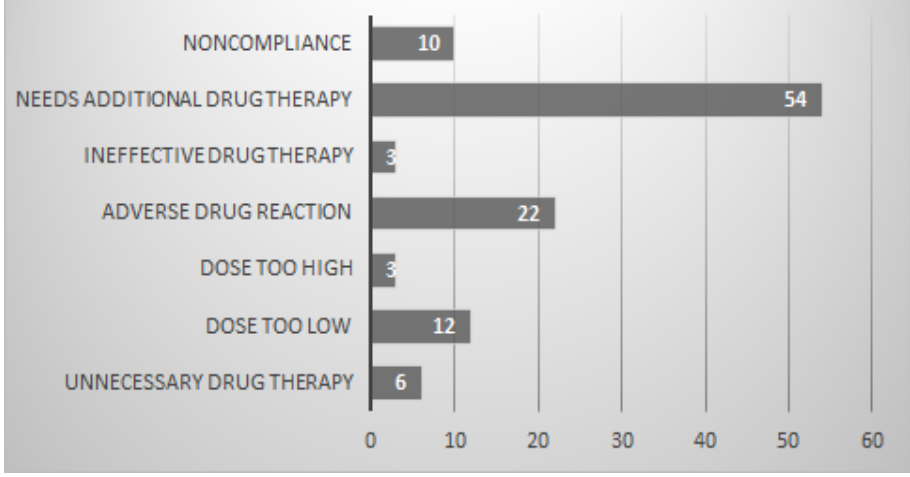

The average number of conditions being treated per patient in this group was 5.7 (range from 1 - 13 conditions per patient). The average number of medications taken per patient in this group was 9.6 (range from 1 - 24 medications per patient). The plotted graph (Figure 2) with total number of medications taken per patient on the $x$-axis and the corresponding total number of MTPs on the $y$-axis does not appear to indicate that there is a correlation between medication burden and burden of medication therapy problems.

\section{Figure 2. Correlation between Medication Burden and Medication Therapy Problems}

\section{Correlation between Medication}

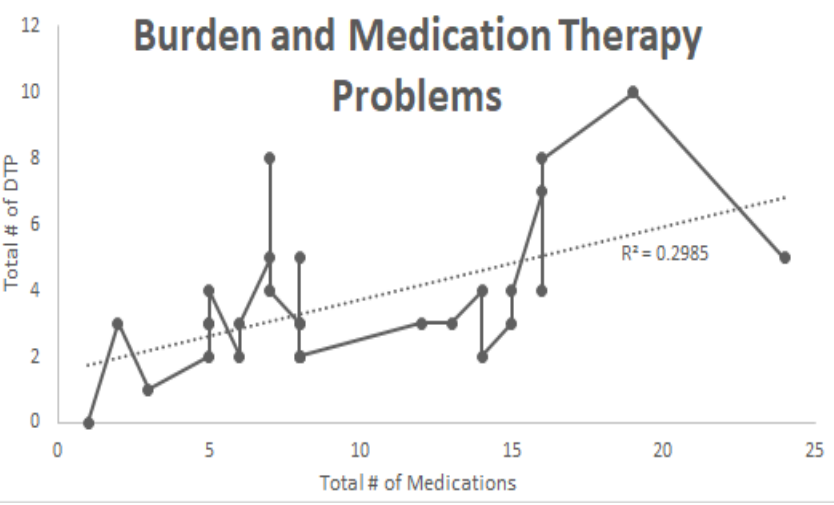

Distribution of MTPs was different when comparing psychiatric medication MTP's to medical MTP's. Psychiatric medications were often associated with a lack of indication and adverse events while medications for medical conditions were associated with lack of effectiveness or the need for additional therapy to reach treatment goals (Figure 3 ).
Figure 3. Distribution of Medication Therapy Problems in Psychiatric and Medical Conditions

\section{Distribution of Medication Therapy Problems in Psychiatric and Medical Conditions}

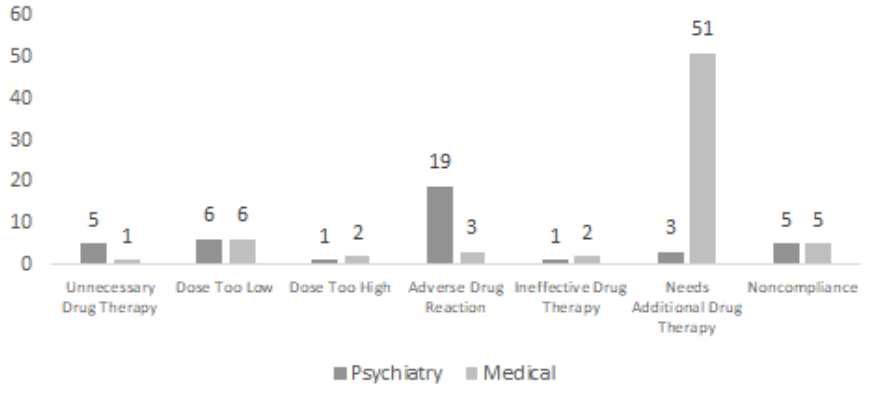

Other incidental findings were the acceptance and value placed on the pharmacist resident by the patients, ACT staff and the chief medical officer psychiatrist.

\section{Discussion}

Adhering to Comprehensive Medication Management and the Pharmacists' Patient Care Process by the Joint Commission of Pharmacy Practitioners ${ }^{18}$ is becoming the community standard of practice for pharmacist provision of direct-patient care services. In its published final form, The Joint Commission of Pharmacy Practitioners expanded the definition of these services: "Medication management services are a spectrum of patientcentered, pharmacist-provided, collaborative services that focus on medication appropriateness, effectiveness, safety, and adherence with the goal of improving health outcomes."

The Assertive Community Treatment (ACT) care model is one of the most widely researched evidence-based practices in community behavioral healthcare for people with $\mathrm{SMI}^{19}$. It has been shown to improve outcomes for people with SMI who are most at-risk for psychiatric crises, hospitalizations, and involvement with the criminal justice system. A multidisciplinary team approach is adopted to provide a comprehensive, assertive treatment approach, which encompasses physical health and behavioral health issues in the SMI population. For standardization of the practice of the model, ACT teams are required to adhere to the ACT fidelity standards developed and refined over the years. In Minnesota, ACT teams adhere to the TMACT (Tool for Measurement of Assertive Community Treatment) developed by Monroe-DeVita and colleagues ${ }^{20}$.

At NPMHC, the clinical pharmacy resident in collaboration with the attending psychiatric physician became the medication expert within the ACT team, monitoring the patients closely and making diagnostic assessments for drug-drug interactions and potential medication side effects, performed therapeutic drug monitoring, assessed organ functions through interpretation of laboratory values, and monitored improvements in the chronic disease states. In so doing, the clinical pharmacist was best positioned to uphold the biomedical fidelity standards nestled in the psychosocial care and ACT framework within a community 
mental health center. Another service included medication/disease state education to the patients and their caregivers. An important and unique role for the clinical pharmacist in the mental health care system, which operations are often siloed, was to serve as the clinical care coordinator and primary care expert to ensure patients' chronic medical conditions and preventative care were optimally managed. The retrospective chart review revealed a high burden of medication therapy problems in this population that was previously not identified by other healthcare professionals involved with the patients' care. This observation further supports findings from existing literature of such health disparity amongst persons with SPMIs.

The statutes governing the fidelity standards for ACT medication management have delegated primary responsibility to the nursing staff. However, in this case and in others it is apparent that the complexity of these patients requires a professional with additional training and skills as demonstrated by other studies ${ }^{4,5,13}$. While nurse provided care may be effective for developing a medication list, arranging medication times, and addressing adherence issues, there is no comprehensive diagnostic assessment of medication therapy problems. Our results support this issue of complexity and the potential missed opportunities to address gaps in actual medication therapy. One of the most striking issues uncovered in this ACT group was the Needs Additional Therapy category, examples of which ranged from immunizations, statin therapy in patients with diabetes, to nicotine replacement therapy for tobacco cessation. Under psychiatric indications, Adverse Drug Reactions was the most common MTP, with notable incidences of extrapyramidal symptoms, metabolic abnormalities, and anticholinergic effects.

Indirect patient care services provided by the clinical pharmacist included formulary management, assistance with prior authorizations, and prescription assistance programs. Regular clinical pharmacist in-services were launched within NPMHC to keep providers abreast of updates in practice, not just in mental health care, but primary care as well.

The ability of ACT staff to meet the patients where they reside in the community setting continues to be the fundamental guiding principle of the ACT care model. With the integration of the pharmacy resident into the ACT team, the ability to deliver some of the clinical patient care services at patients' homes allowed increased accessibility to qualified professionals in the setting of persons disadvantaged by multi-layered socioeconomic factors that plague rural populations. In a survey published by Minnesota Department of Health - Health Economics Program and Office of Rural Health \& Primary Care, results showed insurance coverage disparities decreased across racial groups and between ruralurban areas, however rural Minnesotans were still significantly more likely than their urban counterparts to have difficulties paying healthcare bills and experience greater challenge in getting a timely appointment with primary care providers ${ }^{21}$. Social determinants of mental health, further compounded by barriers in rural geographic areas including limited transportation options, limited broadband internet access, and higher poverty rates - may not seem relevant to clinical pharmacy practice at first glance. However, visits performed at patients' homes allow the clinical pharmacist to adopt a holistic approach to CMM in considerations of actual medication-taking behavior of the patients at home and further allow the clinical pharmacist to provide patient-centered medication recommendations.

One benefit that emerged from incorporating clinical pharmacist services at NPMHC is the improved workload distribution among care team members. The collaborating psychiatric physician reported more efficient clinic flow and being able to serve additional patients due to pre-identified medication therapy problems and recommendations that may be targeted during the psychiatric visits. Other team members reported improved understanding of medications and adverse effects, thereby improved triage and timely communication with pertinent providers.

NPMHC management and clinical staff were so pleased with the results that they subsequently hired the clinical pharmacy resident as their full time clinical pharmacist. The 3-way partnership continues with GuidePoint Pharmacy providing the third year of residency support in collaboration with NPMHC and the College of Pharmacy.

\section{Limitations}

As a retrospective chart review, this study did not include a control group and it also has a small number of patients concentrating on a specific end on a wide spectrum of mental illnesses and severity levels. Therefore, it warrants further investigation to ascertain the landscape of medication therapy problems in other patient populations or non-pharmacist-led CMM.

\section{Conclusion}

The establishment of clinical pharmacy services through a multiorganization partnership mental health focused PGY-1 Pharmacy Residency program within an ACT team in rural Northern Minnesota has been successful on several levels. It has demonstrated the value of CMM provided by a pharmacist in the mental health setting, underscored the enormous value of collaboration and coordination of care on clinical gaps in care, and highlighted the critical importance of professional relationship-building in creating new models of care. These benefits should not be overlooked as new opportunities for pharmacists to expand their roles in mental health and SUDs are pursued. Looking into the future, there are numerous opportunities for pharmacists to make a difference in providing care to rural residents with SMI. Creating interprofessional alliances like the relationships established at NPMHC is the first step in our shared vision of a rural care model. 
Acknowledgements: We thank the leadership at GuidePoint Pharmacy, Northern Pines Mental Health Center, and University of Minnesota for their joint vision and determination for a smooth partnership. We also thank Megan Krueger, PharmD for her expertise and time for resident supervision.

Conflict of Interest: We declare no conflicts of interest or financial interests that the authors or members of their immediate families have in any product or service discussed in the manuscript, including grants (pending or received), employment, gifts, stock holdings or options, honoraria, consultancies, expert testimony, patents and royalties.

\section{Creative Commons License: None}

Disclaimers: The views expressed in the submitted article are of the authors and not an official position of the affiliated institutions.

Funding Sources: The PGY-1 pharmacy resident position is funded by GuidePoint Pharmacy.

Treatment of Human Subjects: IRB exemption granted (ETHOS ID: 152)

\section{References}

1. Bureau of Labor Statistics, Department of Labor, Occupational Outlook Handbook 2010-11 http://bls.gov/oco/.

2. U.S. Department of Health and Human Services (U.S. DHHS 2013). Mental Health: Report to Congress on the Nation's Substance Abuse and Mental Health Workforce Issues, Substance Abuse and Mental Health Services Administration, Center for Mental Health Services, National Institutes of Health, National Institute of Mental Health, 2013.

3. Agency for Healthcare Research and Quality, The Department of Health \& Human Services. (2009). HCUP Facts and Figures: Statistics on Hospital-based Care in the United States, 2009. Retrieved January 16, 2015, from http://www.hcupus.ahrq.gov/reports/factsandfigures/2009/pdfs/FF report 2 009.pdf

4. Schneiderhan ME, Shuster SM, and Davey CS. Twelve-Month Prospective Randomized Study of Pharmacists Utilizing PointOf-Care Testing for Metabolic Syndrome and Related Conditions in Subjects Prescribed Antipsychotics. Prim Care Companion CNS Disord, 2014;15(5):e1-e9.

5. Gable KN, Stunson MJ (Gable et al 2010). Clinical pharmacist intervention on an assertive community treatment team. Community Ment Health J. 2010;46(4): 351-5.

6. Goldstone, L.W., DiPaula, B.A., Caballero, J., Park, S.H., Price, C., Slater, M.Z. (Goldstone et al 2015). Improving medicationrelated outcomes for patients with psychiatric and neurologic disorders: value of psychiatric pharmacists as part of the health care team. The Mental Health Clinician, 5(1):1-28.

7. Bell, S., McLachlan, A.J., Aslani, P., Whitehead, P., Chen, T.F. (2005). Community pharmacy services to optimise the use of medications for mental illness: a systematic review. Australia and New Zealand Health Policy, 2, 29.
8. Bell, J.S., Rosen, A., Aslani, P., Whitehead, P., Chen, T.F. (2007). Developing the role of pharmacists as members of community mental health teams: perspectives of pharmacists and mental health professionals. Research in Social and Administrative Pharmacy, 3(4):392-409.

9. Chen, T.F., Crampton, M., Krass, I., Benrimoj, S.I. (2001). Collaboration between community pharmacists and GPsImpact on interprofessional communication. Journal of Social and Administrative Pharmacy, 18, 83-90.

10. Chen, T.F., De Almeida Neto, A.C. (2007). Exploring elements of interprofessional collaboration between pharmacists and physicians in medication review. Pharmacy World and Science, 29(6), 574-576.

11. Finley, P.R., Rens, H.R., Pont, J.T., Gess, S.L., Louie, C., Bull, S.A., Lee, J.Y., Bero, L.A. (2003). Impact of a collaborative care model on depression in a primary care setting: a randomized controlled trial. Pharmacotherapy, 23(9), 1175-85.

12. Richardson, T.E., O'Reilly, C.L., Chen, T.F. (2014). A comprehensive review of the impact of clinical pharmacy services on patient outcomes in mental health. International Journal of Clinical Pharmacy, 36(2), 222-232.

13. Cobb, C.D. (2014). Optimizing medication use with a pharmacist-provided comprehensive medication management service for patients with psychiatric disorders. Pharmacotherapy, 34(12), 1336-40.

14. Griebe, K.M., Caniff, K.E., Bostwick, J.R. (2016). Psychiatric pharmacists: key allies to improve antipsychotic metabolic monitoring. Psychiatric Services, 67(4), 469-70.

15. Nazarian, P.K., Dopheide, J.A. (2013). Psychiatric pharmacist management of depression in patients with diabetes. Primary Care Companion for CNS Disorders, 15(5).

16. Suehs, B.T., Mican, L.M., Campbell, A.H. (2011). Retrospective evaluation of an inpatient psychiatric pharmacist consultation service. Journal of the American Pharmacists Association, 51(5), 599-604.

17. Tomko, J.R., Ahmed, N., Mukherjee, K., Roma, R.S., Dilucente, D., Orchowski, K. (2013). Evaluation of a discharge medication service on an acute psychiatric unit. Hospital Pharmacy, 48(4), 314-20.

18. Joint Commission of Pharmacy Practitioners (JCPP). Pharmacists' Patient Care Process. May 29, 2014. Available at: https://icpp.net/wpcontent/uploads/2016/03/PatientCareProcess-withsupporting-organizations.pdf.

19. Center for Evidence-Based Practices [Internet]. Ohio: The Center; c1826-Present [cited 2019 Jan 11]. Assertive Community Treatment; [about 1 screen]. Available from: www.centerforebp.case.edu/practices/act

20. Monroe-DeVita, M., Moser, L.L., Teague, G.B. (2013). The tool for measurement of assertive community treatment (TMACT). In M.P. McGovern, G.J. McHugo, R.E. Drake, G.R. Bond, M.R. Merrens. (Eds.), Implementing evidence-based practices in behavioral health. Center City, MN: Hazelden.

21. Minnesota Department of Health (MDH). (2017). Health Care Access in rural Minnesota: results from the Minnesota Health Access Survey. April, 2017. Available at: http://www.health.state.mn.us/divs/orhpc/pubs/2017access .pdf. 